\title{
HACIA LA INSTITUCIONALIDAD DEL SISTEMA ESTATAL DE ARCHIVOS ${ }^{1}$
}

\section{Marcela Barraza Paredes, ${ }^{2}$ Adria Velia González Beltrones, Guadalupe Aleida Valenzuela Miranda ${ }^{3}$}

Sumario: Introducción. II. Panorma evolutivo en México. III. Panorama en Sonora IV. Conclusiones y Propuestas V. Bibliografía y Webgrafía

\section{Resumen}

En el presente documento se sintetiza,el estado del arte del resguardo que hasta nuestros dias se ha realizado en México de los archivos. Tomando en consideración la Declaración Universal sobre los Archivos y la forma de estado adoptada en nuesta Carta Magna (república,democrática,laica y federal) mediante una Ley General se pretende homologar las legislaciones en materia de archivos de 32 entidades federativas EF. Para Sonora, y replicable en las restantes EF se propone la institucionalidad que se concreta a través de la gestión pública que ( Uvalle Berrones:2007,4) es el modo de actuar, decidir, articular capacidades, planes y estrategias para lograr una exitosa gestión de las políticas públicas.En síntesis, el gran reto es confeccionar una Ley Local de Archivos que además de alcanzar una adecuada armonización con lo preceptuado por la Ley General, establezca condiciones para su cumplimiento.

Palabras Clave: Declaración Universal, Archivos,Institucionalidad,

\section{Abstract}

This document summarizes the art state's of the shelter that in ourdays has been made of the archives in Mexico. Taking into consideration the Universal Declaration on the Archives and the form of state adopted in our Constitution (republic, democratic, secular and federal) by means of a General Law it is tried to homologate the 32 federal entities legislations in matter of archives. For Sonora and replicable in the remaining FE, the institutional framework is proposed that is concretized through public management, which, according to Uvalle Berrones, is the way of acting, deciding, articulating capacities, plans and strategies to achieve a successful management of public policies. In summary, the great challenge is to draw up a Local Law of Archives that, in addition to achieving an adequate harmonization with the provisions of the General Law, establishes conditions for its fulfillment.

Key Words: Universal Declaration,Archives, Institutionality

${ }^{1}$ Lo esencial de la propuesta se presentó en el III Encuentro Nacional de Archivos del Poder Ejecutivo en las Entidades Federativas. Archivos y Legislación; Hacia La Implementación de la Ley General de Archivos, UES,UNISON,REA,H Ayuntamiento de Hermosillo Sonora, 25 de junio de 2018.

2 Investigadora e Integrante de la Red Estatal de Archivistas

${ }^{3}$ Docentes Investigadoras adscritas al Departamento de Derecho DCS,URC,UNISON 


\section{Introducción}

El largo compás de espera ${ }^{4}$ del día en el cual México contaría con una Ley General de Archivos LGA, se cumplió, el pasado 15 de junio de 2018, dando con ello, un paso más, en el cumplimiento de la política pública en relación al Sistema Naconal de Archivos. En este tenor, acorde a la única consideración que emite la Comisión de Gobernación de la Cámara de Diputados de la XLIII Legislatura, que a su vez se fundamenta en la Declaración Universal sobre los Archivos, aprobada durante la 36 Reunión de la Conferencia General de la Organización de las Naciones Unidades para la Educación, la Ciencia y la Cultura (UNESCO) tenemos que:

"Los archivos custodian decisiones, actuaciones y memoria. Los archivos conservan un patrimonio único e irremplazable que se transmite de generación en generación. Los documentos son gestionados en los archivos desde su origen para preservar su valor y su significado. Los documentos son fuentes fiables de información que garantizan la seguridad y la transparencia de las actuaciones administrativas. Juegan un papel esencial en el desarrollo de la sociedad contribuyendo a la constitución y salvaguarda de la memoria individual y colectiva. El libre acceso a los archivos enriquece nuestro conocimiento de la sociedad, promueve la democracia, protege los derechos de los ciudadanos y mejora la calidad de vida. ${ }^{5}$

\footnotetext{
${ }^{4}$ Cabe recordar que la expedición de la Ley General de Archivos LGA tiene como fundamento el Decreto por el que se reforman y adicionan diversas disposiciones de la Constitución Política de los Estados Unidos Mexicanos CPEUM, publicado en el DOF el 7 de febrero de 2014, que, entre otras modificaciones, reformó el artículo 6o. y adicionó la fracción XXIX-T al artículo 73,para crear un mecanismo eficiente de acceso a la información y a los documentos contenidos en archivos y para facultar al Congreso de la Unión a expedir "la ley general que establezca la organización y administración homogénea de los archivos en los órdenes federal,estatal,del Distrito Federal y municipal,que determine las bases de organización y funcionamiento del Sistema Nacional de Archivos"

${ }^{5}$ Declaración Universal sobre los archivos, Adoptado por la Asamblea General del Consejo Internacional de Archivos Oslo, Septiembre de 2010 [en línea]Disponible en: https://www.gob.mx/cms/uploads/attachment/file/56206/Declaracion_Universal_sobre_I os_Archivos.pdf
} 
Con esta declaratoria se redimensiona y se eleva el significado de los archivos, por lo tanto, el de su administración y desarrollo; y en esa misma proporción la responsabilidad, sobre todo en los países que han aceptado el compromiso (incluido México) de ejecutar lo necesario en aras de cumplir y mantener la esencia de los archivos con esta nueva perspectiva [transitar de una función netamente administrativa que surge de la gestión y sirve a la gestión administrativa de lo público ( o en su caso de lo privado) a otro de carácter indispensable e insustituible como insumo necesario para ejercer la democracia con el derecho a la verdad y conservación de la memoria por medio del patrimonio documental]

En esta Declaración Universal, el Consejo Internacional de Archivos ICA, por sus siglas en inglés, se reconoce la necesidad de cambios sustanciales como los que se mencionan en el proyecto presentado en Senegal (UNESCO, 2011) enseguida listado

Por ello, nosotros reconocemos

- el carácter único de los archivos como fieles testimonios de las actividades administrativas, culturales e intelectuales y como reflejo de la evolución de las sociedades;

- el carácter esencial de los archivos para garantizar una gestión eficaz, responsable y transparente, para proteger los derechos de los ciudadanos, asegurar la memoria individual y colectiva y para comprender el pasado, documentar el presente para preparar el futuro;

- la diversidad de los archivos para dejar constancia del conjunto de actividades de la humanidad;

- la multiplicidad de soportes en los que los documentos son creados y conservados: papel, audiovisual, digital y otros de cualquier naturaleza;

- el papel de los archiveros, profesionales cualificados, con formación inicial y continuada, sirven a la sociedad garantizando el proceso de producción de los documentos, su selección y su conservación para facilitar su uso;

- la responsabilidad de todos - ciudadanos, gestores y responsables públicos, propietarios y/o custodios de archivos públicos y privados, archiveros y otros profesionales del campo de la información - en la gestión de los archivos.
Por lo tanto nos comprometemos a trabajar conjuntamente para que

- se adopten y se apliquen políticas y normas legales en materia de archivos;

- todos los organismos públicos o privados que producen y utilizan documentos en el ejercicio de sus actividades valoren y ejerzan eficazmente la gestión de sus archivos;

- se doten los recursos adecuados para asegurar la correcta gestión de los archivos, incluyendo profesionales debidamente cualificados;

- los archivos sean gestionados y conservados en condiciones que aseguren su autenticidad, fiabilidad, integridad y uso;

- los archivos sean accesibles a todos, respetando las leyes sobre esta materia y las relativas a los derechos de las personas, de los creadores, de los propietarios y de los usuarios;

- los archivos sean utilizados para contribuir al desarrollo de la responsabilidad de los ciudadanos

El contenido del cuadro anterior nos muestra un panorama de lo que se requiere para iniciar con un cambio hacia la dimensión y concepción de los archivos, que hasta hace 
poco tiempo, a partir de una noción y práctica no adecuada, (vbg considerar el archivar como mera tarea, definida y ejecutada por personal operativo, falto de criterio de institucionalidad) lo que a su vez permitió que los archivos se organizaran de manera aislada y prácticamente bajo criterios individuales con decisión discrecional en su producción, reproducción, guarda y destino, llegando al punto de considerarlos propiedad privada y de allí la discrecionalidad que ya se ha mencionado y que dificulta alcanzar, según menciona Uvalle ${ }^{6}$ la transparencia y la rendición de cuentas ... fórmula para evitar el abuso de los gobernantes en la utilización de los recursos públicos y las decisiones de implementación de políticas públicas ...... los archivos contienen la información documentada que soporta la legalidad, eficacia de las decisiones y acciones de los servidores públicos, por ello son el insumo sustancial de la transparencia, rendición de cuentas y el derecho a la verdad.

\section{Panorama evolutivo en México}

Los archivos en el caso de México, pareciera que han tenido una situación de descuido intencional, dado que los documentos son practicamente la única manera de comprobar de manera fehaciente cualquier acto de corrupción, negligencia o incumplimiento, relacionado con la función de servidor público; pero también tal situación se ha ocasionado por una administración inercial, lugar en el que "solo se hace" "lo que siempre se ha hecho", la formas de trabajo entre los servidores públicos se traspasan de manera operacional; situación que en el caso de los archivos ha sido muy dañina, dado que esa discrecionalidad se ha aplicado en cualquiera de las etapas de la vida de los documentos; desde la producción y reproducción, registro y gestión, archivo de trámite, archivo de concentración y su destino final.

Este problema hasta la fecha, no se ha resuelto, puesto que, a pesar de que se ha emitido leyes y reformas conducentes en materia de transparencia y de archivos, no se ha logrado resolver la ineficacia de las acciones para contar con archivos en las

\footnotetext{
${ }^{6}$ Uvalle Berrones Ricardo,Institucionalidad, eficacia y desafíos de la democracia., 2007,pp.137 [en línea]Disponible en :www.redalyc.org/articulo.oa?id=357535523006
} 
condiciones de administración y organización exigidas por los diferentes formatos, en especial el soporte papel y muy importante el electrónico.

Así tenemos que desde que se publicó la Ley Federal de Transparencia (México. Poder Ejecutivo, Secretaría de Gobernación, 11 de julio de 2002). Se menciona en su artículo 32 la obligación de que los sujetos obligados contarán con archivos organizados para poder soportar su obligación de transparencia. A partir de esa promulgación, cada Estado debió iniciar con la armonizando de sus leyes homólogas para que la política pública de la transparencia se concretara según se menciona en el Plan Nacional de Desarrollo 2000-2006.

..."Este proceso (para profundizar la vida democrática) se acompaña de nuevas formas de estructurar el gobierno, ....... la necesidad de replantear la estructura de la administración pública para imprimirle eficacia, acabar con el burocratismo, terminar con la corrupción y con el dispendio, y de establecer una nueva relación entre gobierno y la sociedad, una relación cimentada en el respeto, la transparencia y la rendición de cuentas..."7 (pág.8)

.." las decisiones discrecionales deben reducirse al mínimo y acotarse y reglamentarse mediante normas claras de orden público, con lo cual se evitarán las oportunidades de corrupción..." (PND2000-2006,pág.45)

Y por último y con relación a los archivos sólo menciona que

..."El Ejecutivo federal asume su responsabilidad de dar acceso a los ciudadanos que lo soliciten a la información de interés público contenida en los documentos elaborados por fuentes oficiales. La actualización del marco jurídico Para ello propondrá la actualización del marco jurídico correspondiente y contribuirá a la modernización de los sistemas con los que operan los archivos que resguardan y catalogan la información elaborada por instancias gubernamentales oficiales."

A partir de lo señalado en los párrafos precitados,aproximadamente, han transcurrido casi 18 años y si bien principalmente, las condiciones jurídicas, han cambiado, puesto que ya se cuenta con una normatividad lo mas cercana,adecuada y tecnicamente

${ }^{7}$ Se encuentra en la sección del mensaje del Presidente de la República [en línea] Disponible en: https://www.gob.mx/cms/uploads/attachment/file/22317/PLAN1.pdf 
posible a la realidad que se pretende regular ( Entre otras, reformas a la Constitución Política de los Estados Unidos Mexicanos, para incluir los temas de Transparencia, rendición de cuentas, anticorrupción y archivos, y su regulación por medio de las leyes ordinarias respectivas, entre ellas la recientemente publicada Ley General de Archivos,

En la realidad fáctica persiste uno de los problemas más urgentes a resolver como lo es la falta de sensibilización sobre el tema, por lo que el reto es generar una cultura respecto a los archivos con esta nueva perspectiva, desde el sector público, como desde el privado, social y ciudadano. En el caso de lo público la instrumentación política, jurídica y administrativa parte de la política pública respectiva y se encamina realmente a cumplir con las expectativas de la Declaración Universal sobre los Archivos, diseñando no solamente la instrumentación jurídica, sino mecanismos que aseguren que se concretará en una acción sistemática, eficiente y permanente; esta permanencia se refiere a que las acciones a realizar vayan encaminadas a propiciar no solo que se cubran las condiciones estructurales y de recursos, sino que la eficiencia sea comprobable tanto en implementación, como en mantenimiento, mejora y desarrollo, dando continuidad a los proyectos, a través de mecanismos sistemáticos, aunados a la interdisciplinariedad necesaria.

En dicha declaración, se explica su esencia y los compromisos que se adquieren con ella, que son referencia de la democracia y la responsabilidad gubernamental y ciudadana para alcanzarlos.

De tal manera que estos fundamentos nos llevan a ubicar a los archivos en un contexto más abierto y de diversificación de sus alcances y por lo mismo de mayor exigencia en su organización y administración. De igual manera nos lleva a ubicar la razón de una política pública de transparencia con base en el derecho a la verdad a través de la transparencia, acceso a la información pública, rendición de cuentas, anticorrupción, gobiernos abiertos, archivos organizados y transparentes.

La democratización de los gobiernos como tendencia internacional, se trata de implementar a través de la política pública que se concreta en acciones normativas como regulador de las acciones de gobierno. 


\section{Panorama Local}

En Sonora se emitió la primer Ley de Transparencia en febrero de 2005, y en ella se regula el tema de los archivos. ..."El Quinto y último Título de la Ley consta, de igual forma que el Título precedente, de un Capítulo Único con idéntica denominación y que es "Del Sistema de Archivos", dentro del cual se integraron disposiciones relacionadas con la obligación de los sujetos obligados oficiales de mantener en operación archivos que permitan localizar con prontitud y seguridad la información pública correspondiente a su función, bajo las condiciones que se especifican en el propio Capítulo. Se establece asimismo que el Instituto expedirá los lineamientos administrativos que los sujetos obligados oficiales deberán implementar para la generación de datos, registros y archivos, así como para la conservación de los mismos. De igual manera, se prevé todo lo relativo al procedimiento de destrucción de los documentos archivados y sus excepciones y se precisa que, cuando alguna unidad administrativa de algún sujeto obligado llegare a desaparecer, los archivos y registros correspondientes deberán ser resguardados por la unidad encargada de su administración.."

En esta misma línea cabe precisar que Sonora ya contaba con su Ley de Archivos intitulada Ley que Regula la Administración de los Documentos Administrativos del Estado de Sonora, publicada desde 1996 con su respectivo Reglamento.La mención de este antecedente tiene la finalidad de observar que a pesar de tener una legislación que obliga a contar con archivos institucionales debidamente organizados y administrados, a la fecha Sonora no ha alcanzado dicho objetivo, ya que en ninguno de los Poderes del Estado de Sonora ni en los 72 gobiernos municipales, se puede constatar la organización de sus archivos, en los términos que la normatividad aplicable lo exige.

${ }^{8}$ Exposición de motivos y la Ley 156 del Estado de Sonora, de Acceso a la Información Pública,reformada el 15 de agosto de 2013[en línea] Disponible en:

http://www.coespo.sonora.gob.mx/documentos/Normatividad/MARCO\%20JURIDICO/ley156accesoinfor macionpublica.pdf 
Si bien en los portales digitales de transparencia se publican los catálogos documentales, de las dependencias y entidades, no son fiables, ya que algunos que se publican no han sido aplicados en la organización y otros están incompletos o diseñados con desconocimiento del producto final.

El punto es que no se cuenta con un documento fiable que constate el avance del Estado de Sonora respecto al cumplimiento de la normativa referente a contar con archivos organizados.

Otro punto sumamente importante, a señalar es la inexistencia, a la fecha, de actas de comité de información donde consten los criterios y los responsables de su aplicación para la valoración de los documentos de archivos, y sin embargo la mayoría de las dependencia y entidades han publicado los propios. Este punto es neurálgico para los archivos, porque una vez que se han destruido los documentos no puede recuperarse la información, a menos que se contara con archivos digitales confiables, y que además cuenten con metadatos o calidad de imagen y organización archivística.

En este orden de ideas,como ha quedado precisado parrafos arriba,desde que se publicó el Plan de Desarrollo 2000-2006, hasta junio de 2018 se logró contar con una Ley General de Archivos. Esta Ley General, cuyo objeto es directamente alcanzar la homologación de los archivos en México a través de una estructura Nacional que se ejecutará a través del Sistema Nacional de Archivos, la que a su vez coordinará a sus homólogos estatales denominados sistemas estatales o locales, y acorde a la Ley, en su artículo 64 en un "marco de respeto de las atribuciones de la federación, entidades federativas, los municipios; así como de las alcaldías de la $\mathrm{Cd}$, de México." Este artículo es un punto importante a tomar en cuenta, porque con esa condición de respeto es la pauta para que las leyes locales pueden establecer las condiciones especiales que se considere requiera cada entidad y sus municipios. Que en teoría podrá llevarse a cabo cuando cada entidad federativa armonice su ley local y archivos, según se exige en el artículo cuarto transitorio citado a continuación : 
"Cuarto. En un plazo de un año, a partir de la entrada en vigor de la presente Ley, las legislaturas de cada entidad federativa, deberán armonizar sus ordenamientos relacionados con la presente Ley";

Si olvidar que esta ley entra en vigor a los 365 días de que se haya publicado, y ese tiempo se cumple el 15 de junio de 2019.

Existen otros tiempos y disposiciones que las entidades federativas deben cumplir, como se muestra en el siguiente cuadro:

\begin{tabular}{|l|l|}
\hline Artículo. Transitorio Décimo Primero & $\begin{array}{l}\text { Los consejo Locales, deberán empezar a } \\
\text { sesionar dentro de los seis meses } \\
\text { posteriores a la adecuación de sus leyes } \\
\text { locales }\end{array}$ \\
\hline Artículo. Transitorio Décimo Cuarto & $\begin{array}{l}\text { Aquellos documentos que se encuentren } \\
\text { en los archivos de concentración y que } \\
\text { antes de la entrada en vigor de la } \\
\text { presente Ley no han sido organizados y } \\
\text { valorados, se les deberá aplicar estos } \\
\text { procesos técnicos, con el objetivo de } \\
\text { identificar el contenido y carácter de su } \\
\text { información y determinar su disposición } \\
\text { documental }\end{array}$ \\
\hline Artículo Transitorio Décimo Séptimo & $\begin{array}{l}\text { En un plazo de un año a partir de la } \\
\text { entrada en vigor de la presente Ley, los } \\
\text { sujetos obligados deberán establecer } \\
\text { programas de capacitación en materia de } \\
\text { gestión documental y administración de } \\
\text { archivos }\end{array}$ \\
\hline
\end{tabular}

Como se puede observar,los tiempos establecido en esta Ley para el cumplimiento de la misma, son muy cortos, tomando en cuenta que en las entidades federativas y haciendo específicamente referencia a Sonora, carece de archivos organizados, personal capacitado o especializado en la materia (en su mayoría) así como la estructura e infraestructura que se requiere para todo lo exigido en la Ley. Además los tiempos políticos y las transiciones administrativas tampoco coadyuvan a la causa, ya que nadie tiene la seguridad de conservar su puesto.

Es decir, no se debe soslayar que en Sonora como en las demás entidades federativas en su caso,tienen impacto los movimientos políticos,ya que la sucesión de Presidente de la República se da posterior a los tres primeros años del gobierno 
estatal en turno y si bien se dispone en teoría de presupuesto, para avanzar en el cumplimiento de las responsabilidades adquiridas en materia archivística, en ocasiones se prioriza la actividad político electoral pasando a segundo término los archivos puesto que no se considera su atención, una prioridad.

De lo precitado en el párrafo inmediato, se puede colegir lo que sucede, pero de manera agravada, en los municipios, por contar con solo tres años de administración [en el primero,la entrega recepción y período en el que se pretende aprender, en el segundo se trata de administrar lo que medianamente se conoce y en el tercero aparecen de nuevo los movimientos para participar en el proceso electoral siguiente] lo que nos lleva a observar la falta de continuidad, ya que si en teoría se hubiese realizado importantes esfuerzos por algunas dependencia y entidades en organizar sus archivos, en los cambios de administración prácticamente se abandonan y dificilmente se retomará dicha actividad por la administración entrante.

Otro factor importante a señalar es la deuda de los municipios y del estado; así como la constante austeridad de la administración. Los archivos, se insiste, hasta ahora, no son prioridad.Para solucionar lo anterior y cumplir con la Ley, sólo se requiere: tiempo, recurso financiero, capital humano y voluntad política para la continuidad de los proyectos una vez que estos hayan sido implementados.

La implementación de este mega proyecto, el de homologar todos los archivos de México, requiere de lograr institucionalizarlos, que para ello, la misma Ley nos habla de una estructura que consiste en un Sistema Nacional de Archivos que observará lo dispuesto en la resoluciones y acuerdos generales que emita el Consejo Nacional de Archivos, que a su vez, estará en coordinación con el Consejo Nacional de Transparencia, y en su momento las entidades federativas replicarán reconociéndose como sistemas locales.

En este tenor, en relación a los Sistemas Locales de Archivo SLA, cabe alertar que si bien la Ley General de Archivos LGA, nos muestra como objeto, la homologación de la organización de todos los archivos institucionales del país, y los particulares de interés general. Para lo cual, la propia LGA preceptúa una estructura (ver esquema 
debajo de estas líneas) que será responsable de que suceda la homologación de manera eficaz y oportuna, paradojicamente, al coordinarse según lo dispuesto por la LGA, con el ámbito estatal, municipal y con los archivos del sector privado, se deja de lado precisamente, al orden federal, invadiendo con ello la soberanía y autonomía del orden local, olvidando, que lo federal no es sinónimo de lo nacional y por lo tanto las autoridades federales, al igual que las locales y municipales deben armonizar en su caso, su legislación en materia de archivos, a lo dispuesto en la precitada Ley General de Archivos $^{9}$ (Ley Marco) y no desaparecer la propia. ${ }^{10}$

Esquema de la relación de los sistemas de archivo

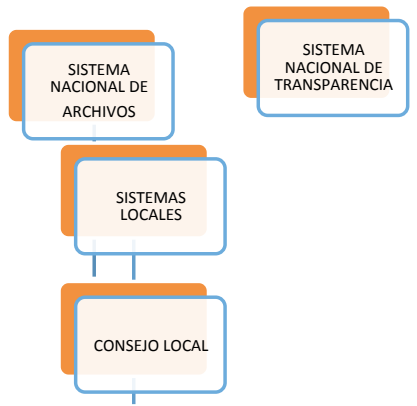

Según lo que nos establece la Ley General de Archivos, en el "Art. 72 los Consejos Locales adoptarán, con carácter obligatorio, en el ámbito de sus respectivas competencias, las determinaciones del Consejo Nacional, dentro de los plazos que éste establezca".

Los Sistemas de Archivo Locales serán el vínculo para que las decisiones y acciones políticas se hagan llegar a los ejecutores que son titulares de la administración pública de los tres poderes y los municipios.

\footnotetext{
${ }^{9}$ ordenamiento tambien aplicable a los particulares en posesión de archivos de interés general.

${ }_{10}^{10}$ Primero. La presente Ley entrará en vigor a los 365 días siguientes contados a partir de su publicación en el Diario Oficial de la Federación.

Segundo. A partir de la entrada en vigor de la Ley se abroga la Ley Federal de Archivos y se derogan todas aquellas disposiciones que contravengan la presente Ley.
} 


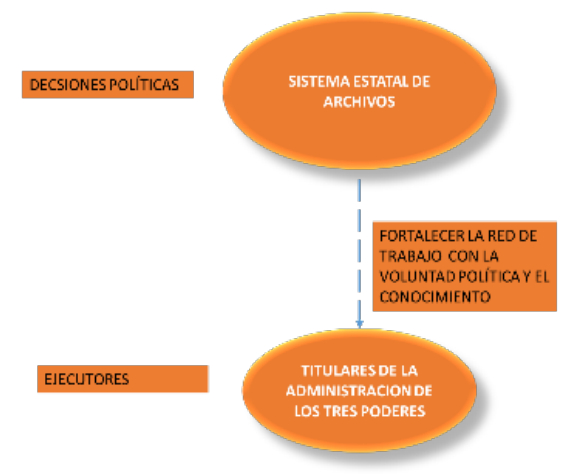

El trabajo que realicen los sistemas locales deberá generar a su vez una red de trabajo con la voluntad política y el conocimiento necesario para alcanzar el objetivo de contar con archivos homologados en las entidades federativas.

Si bien la LGA,otorga un nuevo y obligado estatus orgánico a quienes administrarán el proceso, por ejemplo los titulares del Archivo General ${ }^{11}$ tendrán nivel Subsecretario y en el caso de las dependencias y entidades será un Coordinador con nivel de dirección general o su equivalente. Esto realmente es algo nuevo para una función a la que no se le había reconocido tal carácter y se venía ejerciendo a nivel Dirección de Area y/o nivel de Jefatura de Departamento. En adelante el Coordinador tendrá responsables de archivos por cada área, creando así el Sistema Institucional para la Administración de Archivos.

${ }^{11} \mathrm{Al}$ igual que en el caso de la Auditoría Superior de la Federación ASF no se le otorgó al Archivo General de la Nación el carácter de Órgano Autónomo, aun cuando de manera tácita y en algunos preceptos de manera expresa en la CPEUM y en disposiciones de ella derivadas, se les otorga facultades como órganos nacionales y no solo de carácter federal (pues no obstante la autonomía técnica a ellos otorgada sigue siendo en el primer caso un órgano dependiente de una sección del Poder Legislativo---la Cámara de Diputados--- y el segundo un órgano descentralizado dependiente del Poder Ejecutivo Federal (LGA Artículo 104. El Archivo General es un organismo descentralizado no sectorizado, con personalidad jurídica y patrimonio propio, con autonomía técnica y de gestión para el cumplimiento de sus atribuciones, objeto y fines; su domicilio legal es en la Ciudad de México. 


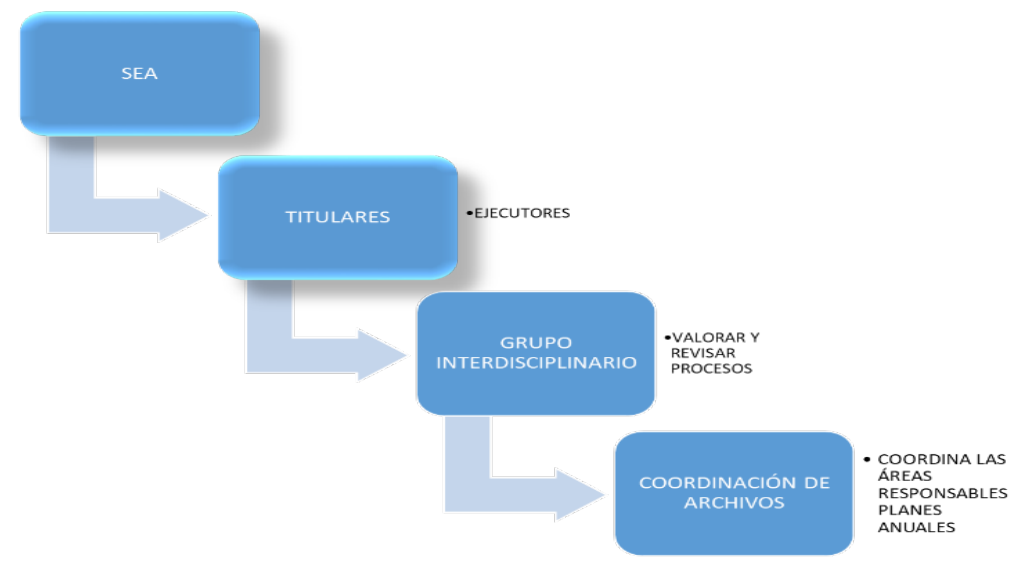

Si se logra implementar respetando lo establecido en la ley y obteniendo los resultados del objeto de la misma, solo entonces podremos considerar que el Sistema Local de Archivos ha alcanzado a institucionalizarse, al punto de llevar la implementación de la política pública a lo procedimental que es la última parte donde se concretiza, es decir en la operación que "... garanticen su efectividad en términos de gobierno. ...Los procedimientos se relacionan con la existencia de organizaciones -expresión material de las instituciones (Uvalle:2007 p.140) que atiende formalidades, tiempos, reglas, autorizaciones, delimitan funciones, dirigen a la evidencia, controlan acciones y actitudes. Los procedimientos tienen como objetivo ser eficaces en el ejercicio de sus funciones para alcanzar el objeto para lo que fueron creadas las instituciones.

Toda esa promoción de eficacia que se realiza con las reglas procedimentales tiene como motor la gestión pública donde las instituciones y la institucionalidad son el ámbito donde se ejerce. "el gobierno de lo público significa el gobierno por políticas y estás se inscriben en la gestión pública porque es la responsable de ordenar, articular, elaborar, implementar y evaluar las acciones del gobierno"12

Los Sistemas Locales de Archivo tendrán la facultad de asegurarse que en las entidades federativas se implemente la homologación de los archivos que es el objeto de la Ley, facultades que se establecen en el artículo 73 :

\footnotetext{
${ }^{12}$ Uvalle Berrones, Ricardo.Los fundamentos institucionales de la gestión pública. VII Congreso internacional del CLAD. En Lisboa : 2002,pp.2[en línea] Disponible en:http://unpan1.un.org/intradoc/groups/public/documents/CLAD/clad0043511.pdf
} 
I. Implementar las políticas, programas, lineamientos y directrices para la organización y administración de los archivos que establezca el Consejo Nacional;

II. Aprobar criterios para homologar la organización y conservación de los archivos locales;

III. Aprobar las campañas de difusión sobre la importancia de los archivos como fuente de información esencial y como parte de la memoria colectiva;

IV. En el marco del Consejo Nacional, los Consejos Locales podrán proponer las disposiciones que regulen la creación y uso de sistemas automatizados, para la gestión documental y administración de archivos para los sujetos obligados del ámbito local, que contribuyan a la organización y conservación homogénea de sus archivos;

V. Establecer mecanismos de coordinación con los sujetos obligados de los municipios o de las alcaldías, según corresponda;

VI. Operar como mecanismo de enlace y coordinación con el Consejo Nacional; VII. Fomentar la generación, uso y distribución de datos en formatos abiertos, y VIII. Las demás establecidas en esta Ley.

Y las obligaciones para los sujetos obligados, según los artículos 10 y 11 de la Ley:

I. Administrar, organizar, y conservar de manera homogénea los documentos de archivo que produzcan, reciban, obtengan, adquieran, transformen o posean, de acuerdo con sus facultades, competencias, atribuciones o funciones, los estándares y principios en materia archivística, los términos de esta Ley y demás disposiciones jurídicas que les sean aplicables;

II. Establecer un sistema institucional para la administración de sus archivos y llevar a cabo los procesos de gestión documental;

III. Integrar los documentos en expedientes;

IV. Inscribir en el Registro Nacional la existencia y ubicación de archivos bajo su resguardo;

V. Conformar un grupo interdisciplinario en términos de las disposiciones reglamentarias, que coadyuve en la valoración documental;

VI. Dotar a los documentos de archivo de los elementos de identificación necesarios para asegurar que mantengan su procedencia y orden original;

VII. Destinar los espacios y equipos necesarios para el funcionamiento de sus archivos;

VIII. Promover el desarrollo de infraestructura y equipamiento para la gestión documental y administración de archivos;

IX. Racionalizar la producción, uso, distribución y control de los documentos de archivo;

$X$. Resguardar los documentos contenidos en sus archivos;

$\mathrm{XI}$. Aplicar métodos y medidas para la organización, protección y conservación de los documentos de archivo, considerando el estado que guardan y el espacio para su almacenamiento; así como procurar el resguardo digital de dichos documentos, de conformidad con esta Ley y las demás disposiciones jurídicas aplicables, y

XII. Las demás disposiciones establecidas en esta Ley y otras disposiciones jurídicas aplicables. 
Entre las atribuciones del Sistema Local y las obligaciones de los Sujetos Obligados, la vigilancia del cumplimiento de la Ley es a cargo de los órganos Internos de Control, que acorde a lo dispuesto en el artículo 12 (segundo párrafo) ..." Los órganos internos de control y sus homólogos en la federación y las entidades federativas, vigilarán el estricto cumplimiento de la presente Ley, de acuerdo con sus competencias e integrarán auditorías archivísticas en sus programas anuales de trabajo"

Que el Estado de Sonora cumpla con la Ley Estatal de Archivos,significa que ello comprende todo los archivos del poder ejecutivo, judicial y ejecutivo, 72 municipios de los cuales sólo ocho de ellos sobrepasan los 70,000 habitantes, (INEGI.2015) ${ }^{13}$; además de los órganos autónomos, partidos políticos, fideicomisos y fondos públicos, así como de cualquier persona física, moral o sindicato que reciba y ejerza recursos públicos o realice actos de autoridad federal o estatal, lo cual se traduce en es un universo muy amplio y distribuido en un territorio muy grande.

Lo que a su vez implica un gran esfuerzo y una gran cantidad de recursos, ya que los órganos internos de control deberán contar con personal preparado para las auditorías archivística y la parte de fondo como la calidad técnica o especializada del proceso tendrá tambien que cubrirse.

La obligación de órgano interno de control se limita a vigilar el proceso de cada uno de los sujetos obligados, pero previo a ello cada uno debe contar con un diagnóstico de las condiciones de los archivos y determinar su estado real para proponer estrategias a nivel estado y acciones efectivas no sólo para gestionar recursos, sino para identificar los puntos de riesgo, ya que los proyectos que cada sujeto elaborará será a la medida de sus condiciones y necesidades, pero el interés común es el mismo, lograr homologar sus sistemas de archivo acorde a la normatividad.

Se trata de institucionalizar una función transversal que deberá implementarse por medio de una planeación estratégica que tomando los tiempos tan cortos que impone

\footnotetext{
${ }^{13}$ INEGI, 2015[en línea] Disponible en: http://cuentame.inegi.org.mx/monografias/informacion/son/poblacion/
} 
la Ley para su cumplimiento, se avizora como un reto difícil de lograr, ya que acorde a lo dispuesto en los artículos transitorios:

Décimo. Los Consejos Locales, deberán empezar a sesionar dentro de los seis meses posteriores a la adecuación de sus leyes locales.

Décimo Primero. Los sujetos obligados deberán implementar su sistema institucional, dentro de los seis meses posteriores a la entrada en vigor de la presente Ley.

Décimo Cuarto. Aquellos documentos que se encuentren en los archivos de concentración y que antes de la entrada en vigor de la presente Ley no han sido organizados y valorados, se les deberá aplicar estos procesos técnicos archivísticos, con el objetivo de identificar el contenido y carácter de la información determinar su disposición documental.

\section{Conclusiones y Propuestas}

Por lo expuesto se considera que para asegurar el institucionalizar la homogenización de los archivos, es necesario que se prevea desde el Sistema Estatal de Archivos:

Contar con un grupo de trabajo técnico dentro del Sistema Estatal de Archivo que planifique, coordine y ejecute el seguimiento de la implementación del proyecto de homologación de los archivos. Que será el que trabaje coordinadamente con los archivos generales, los órganos internos de control y municipios, otorgándole al Sistema la atribución de vigilancia de eficiencia del cumplimiento técnico.

Elaborar un diagnóstico general que dé el panorama de la condición de los archivos públicos de Sonora y dar seguimiento al cumplimiento de metas e indicadores que permita ver el avance como estado

Realizar un inventario de todos los archivos del Estado de Sonora a través de sus Archivo Generales y los sujetos obligados que no dependan de uno y los privados.

Elaborar un Reglamento del Sistema y gestionar su publicación. 
Crear y sustentar una página de transparencia para conocer el estado inicial y las acciones y resultados obtenidos; así como la gestión y uso de los recursos.

Generar estrategias para asegurar la continuidad de los proyectos a pesar de los tiempos políticos que han frenado el desarrollo de los archivos en la administración pública.

Estamos conscientes que llevar a cabo un proceso de institucionalización de sistemas de archivo, homólogos en el Estado de Sonora, ${ }^{14}$ no es una tarea fácil ni a corto plazo como lo dispone y obliga la ley, en cuanto a estar preparados, con sistemas institucionales de archivos en todo el estado, pero el plazo ya comenzó su marcha.

${ }^{14}$ aún si se contara con los elementos necesarios y que se engranaran de manera eficaz y sin tropiezos. 


\section{Bibliografía y Webgrafía}

Declaración Universal sobre los archivos, Adoptada por la Asamblea General del Consejo Internacional de Archivos Oslo, Septiembre de 2010 [en línea]Disponible en: https://www.gob.mx/cms/uploads/attachment/file/56206/Declaracion_Universal_sobre_los_Archivos.pdf

Instituto Nacional de Geografía y EstadísticalNEGI. 2015,[en líne] Disponible en: http://cuentame.inegi.org.mx/monografias/informacion/son/poblacion/

Uvalle Berrones Ricardo,Institucionalidad, eficacia y desafíos de la democracia., 2007,pp.137 [en línea]Disponible en :www.redalyc.org/articulo.oa?id=357535523006

Los fundamentos institucionales de la gestión pública. VII Congreso internacional del CLAD. En Lisboa : 2002,pp.2[en línea] Disponible en:http://unpan1.un.org/intradoc/groups/public/documents/CLAD/clad0043511.pdf

\section{Legislación}

Ley General de Archivos, Nueva Ley publicada en el Diario Oficial de la Federación el 15 de junio de 2018, Texto Vigente a partir del 15-06-2019

Fecha de recepción 24/03/2018

Fecha de aceptación 30/06/2018 